T. E. Cecil

Nagoya Math. J.

Vol. 55 (1974), 5-31

\title{
GEOMETRIC APPLICATIONS OF CRITICAL POINT THEORY TO SUBMANIFOLDS OF COMPLEX PROJECTIVE SPACE
}

\author{
THOMAS E. CECIL
}

\section{Section 0-Introduction.}

In a recent paper, [6], Nomizu and Rodriguez found a geometric characterization of umbilical submanifolds $M^{n} \subset \boldsymbol{R}^{n+p}$ in terms of the critical point behavior of a certain class of functions $L_{p}, p \in \boldsymbol{R}^{n+p}$, on $M^{n}$. In that case, if $p \in \boldsymbol{R}^{n+p}, x \in M^{n}$, then $L_{p}(x)=(d(x, p))^{2}$, where $d$ is the Euclidean distance function.

The result of Nomizu and Rodriguez can be expressed as follows. Let $M^{n}(n \geqslant 2)$ be a connected, complete Riemannian manifold isometrically immersed in $\boldsymbol{R}^{n+p}$. Suppose there exists a dense subset $D$ on $\boldsymbol{R}^{n+p}$ such that every function of the form $L_{p}, p \in D$, has index 0 or $n$ at any of its non-degenerate critical points. Then $M^{n}$ is an umbilical submanifold, that is $M^{n}$ is embedded in $\boldsymbol{R}^{n+p}$ as a Euclidean subspace, $\boldsymbol{R}^{n}$, or a Euclidean $n$-sphere, $S^{n}$.

Since the set of all points $p \in \boldsymbol{R}^{n+p}$ such that $L_{p}$ is a Morse function is a dense subset of $\boldsymbol{R}^{n+p}$, the above theorem could also have been stated in terms of Morse functions of the form $L_{p}$.

In this paper, we prove results analogous to those of Nomizu and Rodriguez for submanifolds of complex projective space, $P^{m}(C)$, endowed with the standard Fubini-Study metric.

Let $M^{n}$ be a complex $n$-dimensional submanifold of $P^{n+p}(C)$. For $p \in P^{n+p}(C), x \in M^{n}$, the function $L_{p}(x)$ which we define is essentially the distance in $P^{n+p}(C)$ from $p$ to $x$. In section 2, we define the concept of a focal point of $\left(M^{n}, x\right)$. We then prove an Index Theorem for $L_{p}$ which states that the index of $L_{p}$ at a non-degenerate critical point $x$ is equal to the number of focal points of $\left(M^{n}, x\right)$ on the geodesic in $P^{n+p}(C)$ from $x$ to $p$.

Received July 9, 1973.

This research was partially supported by NSF grant GP 28419 A1. 
In the process, we find that if $L_{p}(x)=\pi / 2$, then $L_{p}$ has a degenerate critical point at $x$. Because of this, it is impossible to state the following result in terms of Morse functions of the form $L_{p}$.

Our main result is the following. Let $M^{n}(n \geqslant 2)$ be a connected, complete, complex $n$-dimensional Kählerian manifold which is holomorphically and isometrically immersed in $P^{n+p}(C)$. Assume there exists a dense subset $D$ of $P^{n+p}(C)$ such that every function of the form $L_{p}, p \in D$, has index 0 or $n$ at any of its non-degenerate critical points. Then $M^{n}$ is $P^{n}(C)$ or $Q^{n}(C)$. Here $P^{n}(C)$ denotes a totally geodesic submanifold of $P^{n+p}(C)$, and $Q^{n}(C)$ is the standard complex quadric hypersurface of a totally geodesic $P^{n+1}(C) \subset P^{n+p}(C)$.

In section 3 , we prove the above result for co-dimension $p=1$; and in section 4 , we extend the result to arbitrary co-dimensions. Section 5 is devoted to a detailed study of the interesting special case $Q^{n}(C)$ $\subset P^{n+1}(C)$. We find, among other things, that the set of focal points is $P^{n+1}(R)$, a real $(n+1)$-dimensional projective space naturally embedded in $P^{n+1}(C)$.

The author would like to express his sincere gratitude to his adviser, Katsumi Nomizu, for his assistance in his work.

\section{Section 1-Preliminaries.}

We first recall the construction of the Fubini-Study metric on $P^{m}(C)$ (see [4], vol. II, p. 273-78 and [7], p. 514-515, for more detail). We consider $P^{m}(C)$ endowed with the Fubini-Study metric of constant holomorphic sectional curvature 4 (we choose 4 instead of 1 for the curvature to make calculations easier).

Consider $C^{m+1}$ with natural basis $e_{0}, \cdots, e_{m}$. The natural Hermitian inner product on $C^{m+1}$ is defined by

$$
(z, w)=\sum_{k=0}^{m} z^{k} \bar{w}^{k}
$$

where

$$
z=\sum_{k=0}^{m} z^{k} e_{k} \quad \text { and } \quad w=\sum_{k=0}^{m} w^{k} e_{k}
$$

The Euclidean metric $g$ on $C^{m+1}$ is given by

$$
g(z, w)=\operatorname{Re}(z, w) \quad \text { for } z, w \in C^{m+1} .
$$


The unit sphere

$$
S^{2 m+1}=\left\{z \in C^{m+1} \mid(z, z)=1\right\}
$$

is a principal fibre bundle over $P^{m}(C)$ with structure group $S^{1}$ and projection $\pi$. With the natural identification between vectors tangent to $S^{2 m+1}$ and vectors in $C^{m+1}$, one can show that for $z \in S^{2 m+1}$, the tangent space to $S^{2 m+1}$ at $z$, which we denote as $T_{z}\left(S^{2 m+1}\right)$, is given by

$$
T_{z}\left(S^{2 m+1}\right)=\left\{w \in C^{m+1} \mid g(z, w)=0\right\} .
$$

If we define $T_{z}^{\prime}$ by

$$
T_{z}^{\prime}=\left\{w \in C^{m+1} \mid g(z, w)=g(i z, w)=0\right\},
$$

then $T_{z}^{\prime}$ is a subspace of $T_{z}\left(S^{2 m+1}\right)$ whose orthogonal complement is $\{i z\}$, the 1-dimensional subspace spanned by the vector $i z$. The distribution $T^{\prime}$ defines a connection in the principal fibre bundle $S^{2 m+1}\left(P^{m}(C), S^{1}\right)$, in that $T_{z}^{\prime}$ is complementary to the subspace $\{i z\}$ tangent to the fibre through $z$, and $T^{\prime}$ is invariant by the action of $S^{1}$. Thus the projection $\pi$ induces a linear isomorphism $\pi_{*}$ of $T_{z}^{\prime}$ onto $T_{\pi(z)}\left(P^{m}(C)\right)$, and $\pi_{*}$ maps $\{i z\}$ into 0 for each $z \in S^{2 m+1}$.

We define the Fubini-Study metric, $\tilde{g}$, of constant holomorphic sectional curvature 4 by the equation

$$
\tilde{g}(X, Y)=g\left(X^{\prime}, Y^{\prime}\right)
$$

where $X, Y \in T_{p}\left(P^{m}(C)\right)$ and $X^{\prime}, Y^{\prime}$ are their respective horizontal lifts at $z$ where $\pi(z)=p$. Since $g$ is invariant by the action of $S^{1}$, the definition is independent of the choice of $z$. The complex structure on $T_{z}^{\prime}$ defined by multiplication by $i$ induces the canonical complex structure, $J$, on $P^{m}(C)$ by means of the isomorphism $\pi_{*}$. Finally, $\pi_{*}$ induces the Kählerian connection, $\tilde{\nabla}$, on $P^{m}(C)$ in the following way. Let $X, Y$ be vector fields on $P^{m}(C)$, and let $X^{\prime}, Y^{\prime}$ be their respective horizontal lifts. Then for $\nabla^{\prime}$ the covariant derivative on $S^{2 m+1}$, the equation

$$
\tilde{\nabla}_{X} Y=\pi_{*}\left(\nabla_{X^{\prime}}^{\prime} Y^{\prime}\right)
$$

defines the Kählerian connection on $P^{m}(C)$.

Section 2-Focal points, the functions $L_{p}$, and the Index Theorem.

Let $M^{n}$ be a connected, complex $n$-dimensional Kählerian manifold, and let $f$ be a holomorphic and isometric immersion of $M^{n}$ into $P^{n+p}(C)$. 
Let $N\left(M^{n}\right)$ denote the normal bundle of $M^{n}$. Any point of $N\left(M^{n}\right)$ can be represented by a pair $(x, r \xi)$ where $x \in M^{n}, r \in R$, and $\xi$ is a unitlength vector in $T_{x}^{\perp}\left(M^{n}\right)$, the normal space to $M^{n}$ at $f(x)$.

We define $\gamma(x, \xi, r),-\infty<r<\infty$, to be the geodesic in $P^{n+p}(C)$ parametrized by arc-length parameter $r$ such that

$$
\gamma(x, \xi, 0)=f(x) \text { and } \vec{\gamma}(x, \xi, 0)=\xi .
$$

In terms of the vector representation of $P^{n+p}(C), \gamma(x, \xi, r)$ can be described as follows. Let $w \in S^{2(n+p)+1}$ such that $\pi(w)=f(x)$, and let $\xi^{\prime} \in T_{w}^{\prime}$ such that $\pi_{*}\left(\xi^{\prime}\right)=\xi$. Then

$$
r(x, \xi, r)=\pi\left(\cos r w+\sin r \xi^{\prime}\right) .
$$

Of course, $\gamma(x, \xi, r)$ does not depend on the choice of $w$.

We define a map $F: N\left(M^{n}\right) \rightarrow P^{n+p}(C)$ by

$$
F(x, r \xi)=\gamma(x, \xi, r) \text {. }
$$

We note that for any values of $x, \xi$ and $r$ the following holds,

$$
F(x,(r+\pi) \xi)=F(x, r \xi) .
$$

Thus we may restrict the range of values of $r$ to $-\pi / 2 \leq r \leq \pi / 2$.

For $\xi \in T_{\bar{x}}\left(M^{n}\right)$, let $A_{\xi}$ denote the symmetric endomorphism of $T_{x}\left(M^{n}\right)$ corresponding to the second fundamental form of $M^{n}$ at $x$ in the direction of $\xi$. We first prove the following proposition.

Proposition 1. Let $(x, r \xi) \in N\left(M^{n}\right)$. Then $F_{*}$, the Jacobian of $F$, is degenerate at $(x, r \xi)$ in precisely the following cases:

(i) If $r= \pm \pi / 2$, then $F_{*}$ is degenerate.

(ii) For $-\pi / 2 \leq r \leq \pi / 2$, there is a contribution of $\nu>0$ to the nullity of $F_{*}$ at $(x, r \xi)$ if

$$
\cot r=k
$$

where $k$ is an eigen-value of multiplicity $\nu$ of $A_{\xi}$.

Proof. Fix the point $(x, r \xi) \in N\left(M^{n}\right)$; we want to examine the nullity of $F_{*}$ at $(x, r \xi)$. We assume for the moment that $r \neq 0$, and by replacing $\xi$ by $-\xi$ if necessary, we may assume $r>0$.

Let $U$ be a local co-ordinate neighborhood of $x$ in $M^{n}$ with local coordinates $u^{1}, u^{2}, \cdots, u^{2 n}$. Choose orthonormal normal vector fields $\xi_{1}, \cdots$, 
$\xi_{p}, J \xi_{1}, \cdots, J \xi_{p}$ on $U$ such that $\xi_{1}(x)=\xi$. For ease in notation, we let $\xi_{p+j}=J \xi_{j}$ for $1 \leq j \leq p$. For $u \in U, \eta \in T \frac{\perp}{u}\left(M^{n}\right)$, we can write

$$
\eta=\mu\left(\left(1-\sum_{j=2}^{2 p}\left(t^{j}\right)^{2}\right)^{1 / 2} \xi_{1}+t^{2} \xi_{2}+\cdots+t^{2 p} \xi_{2 p}\right)
$$

where $0 \leq \mu<\infty, 0 \leq\left|t^{j}\right| \leq 1$ for all $j$, and $\sum_{j=2}^{2 p}\left(t^{j}\right)^{2} \leq 1$. The $t^{j}$ are the direction cosines of $\eta$ and $\mu=\|\eta\|$. The co-ordinates $u^{1}, \cdots, u^{2 n}, \mu, t^{2}$, $\cdots, t^{2 p}$ are local co-ordinates for $N(U)$.

Let $w \in S^{2(n+p)+1}$. To avoid confusion, we will denote the map $\pi_{*}: T_{w}^{\prime} \rightarrow T_{\pi(w)}\left(P^{n+p}(C)\right)$ by $\left(\pi_{*}\right)_{w}$ when such precision is required.

Now let $w \in S^{2(n+p)+1}$ such that $\pi(w)=f(x)$. We define $z \in S^{2(n+p)+1}$ by the vector equation

$$
z=\cos r w+\sin r \xi^{\prime}
$$

where $\left(\pi_{*}\right)_{w}\left(\xi^{\prime}\right)=\xi$. Then $F(x, r \xi)=\pi(z)$. For any $j, 2 \leq j \leq 2 p$, the definition of $F$ implies that

$$
\left.F_{*}\left(\frac{\partial}{\partial t^{j}}\right)\right|_{(x, r \xi)}=\left.\left(\pi_{*}\right)_{z}\left(\vec{\eta}\left(t^{j}\right)\right)\right|_{t^{j}=0}
$$

where $\eta\left(t^{j}\right)$ is a curve on $S^{2(n+p)+1}$ defined by

$$
\eta\left(t^{j}\right)=\cos r w+\sin r\left(\left(1-\left(t^{j}\right)^{2}\right)^{1 / 2} \xi_{1}^{\prime}+t^{j} \xi_{j}^{\prime}\right),
$$

where $\xi_{1}^{\prime}, \xi_{j}^{i}$ are the horizontal lifts of $\xi_{1}, \xi_{j}$ respectively to $T_{w}^{\prime}$. We see that $\eta(0)=z$ for any $j$.

If $r= \pm \pi / 2$, we will show $\left.F_{*}\left(\partial / \partial t^{p+1}\right)\right|_{(x, r \xi)}=0$. In that case, $\xi_{p+1}=$ $J \xi_{1}$ and for $r=\pi / 2$

$$
\left.\vec{\eta}\left(t^{p+1}\right)\right|_{t^{p+1}=0}=i \eta(0)=i z
$$

and

$$
\left.F_{*}\left(\frac{\partial}{\partial t^{p+1}}\right)\right|_{(x, r \xi)}=\left(\pi_{*}\right)_{z}(i z)=0
$$

The case $r=-\pi / 2$ is handled similarly. This proves (i).

For $|r|<\pi / 2$, a straight-forward calculation which we omit shows,

$$
\left.F_{*}\left(\frac{\partial}{\partial t^{j}}\right)\right|_{(x, r \xi)}=\sin r\left(\pi_{*}\right)_{z}\left(\xi_{j}^{\prime}\right) \neq 0, \quad \text { for } 2 \leq j \leq 2 p
$$

and 


$$
\left.F_{*}\left(\frac{\partial}{\partial \mu}\right)\right|_{(x, r \xi)}=\left(\pi_{*}\right)_{z}\left(\sin r w+\cos r \xi_{1}^{\prime}\right) \neq 0
$$

In fact, these computations show that if

$$
V=a_{1}\left(\frac{\partial}{\partial \mu}\right)+\sum_{j=2}^{2 p} a_{j}\left(\frac{\partial}{\partial t^{j}}\right) \in T_{(x, r \xi)}(N(U)),
$$

then $F_{*}(V)=0$ only if $a_{j}=0$ for all $j$. If we let

$$
X=\sum_{j=1}^{2 n} b_{j}\left(\frac{\partial}{\partial u^{j}}\right) \in T_{(x, r \xi)}(N(U))
$$

we shall next compute $F_{*}(X)$. That computation and the above will show that

$$
F_{*}(X+V)=0 \quad \text { only if } V=0 \text {. }
$$

(We remark that if $r=0$, we must choose a slightly different co-ordinate system to obtain the same result.)

Consider a vector $X=\sum_{j=1}^{2 n} b_{j}\left(\partial / \partial u^{j}\right) \in T_{(x, r \xi)}(N(U))$. If $r=0$, one easily shows $F_{*}(X)=X$ and so $F_{*}$ is non-degenerate at $(x, 0)$. Assume again, then, that $r>0$. Considering $T_{(x, r \xi)}(N(U))$ as $T_{x}(U) \oplus \boldsymbol{R}^{2 p}$, we can write $X=(Y, 0)$ where $Y \in T_{x}(U)$. To facilitate the computation of $F_{*}(X)$, we assume that the vector field $\xi_{1}$ defined above has been chosen so that

$$
\nabla_{\bar{Y}}^{\frac{1}{Y}} \xi_{1}=0
$$

where $\nabla^{\perp}$ is the connection in the normal bundle.

Locally, i.e. for some $\varepsilon>0$, there is a curve $\beta(t),-\varepsilon<t<\varepsilon$, in $M^{n}$ such that $\beta(0)=x$ and $\vec{\beta}(0)=Y$. Let $\alpha(t)$ be the lift of $\beta(t)$ to $S^{2(n+p)+1}$ so that $\alpha(0)=w$, and $\pi(\alpha(t))=f(\beta(t))$ for $-\varepsilon<t<\varepsilon$.

If we define the curve $\eta(t)$ in $S^{2(n+p)+1}$ by

$$
\eta(t)=\cos r \alpha(t)+\sin r \xi_{1}^{\prime}(\alpha(t))
$$

then $\eta(0)=z$, and

$$
F_{*}(X)=\left(\pi_{*}\right)_{z}(\vec{\eta}(0))
$$

We need to find the component of $\vec{\eta}(0)$ in $T_{z}^{\prime}$. Considering $\eta(t)$ as a curve in $C^{n+p+1}$, we find

$$
\vec{\eta}(t)=\cos r \vec{\alpha}(t)+\sin r D_{\vec{\alpha}(t)} \xi_{1}^{\prime}
$$


where $D$ is the Euclidean covariant derivative in $C^{n+p+1}$. Since $g(\vec{\alpha}(t)$, $\xi_{1}^{\prime}(\alpha(t))=0$ for $-\varepsilon<t<\varepsilon$, we have $D_{\vec{\alpha}(t)} \xi_{1}^{\prime}=\nabla_{\vec{\alpha}(t)}^{\prime} \xi_{1}^{\prime}$. Thus we have by evaluating (2) at $t=0$,

$$
\vec{\eta}(0)=\cos r \vec{\alpha}(0)+\sin r \nabla_{\vec{\alpha}(0)}^{\prime} \xi_{1}^{\prime} .
$$

One can show by a straight-forward calculation that

$$
g(\vec{\eta}(0), z)=0=g(\vec{\eta}(0), i z),
$$

and hence $\vec{\eta}(0) \in T_{z}^{\prime}$. Since $\left(\pi_{*}\right)$ is an isomorphism on $T_{z}^{\prime}$, we have shown

$$
\left(\pi_{*}\right)_{z} \vec{\eta}(0)=0 \quad \text { if and only if } \vec{\eta}(0)=0 \text {. }
$$

To find when $\vec{\eta}(0)=0$, we proceed as follows. We displace the vector $\vec{\eta}(0) \in T_{z}^{\prime}$ by Euclidean parallelism and consider $\vec{\eta}(0) \in T_{w}\left(S^{2(n+p)+1}\right)$. Equation (3) shows that, in fact, $\vec{\eta}(0) \in T_{w}^{\prime}$ since $\vec{\alpha}(t)$ and $\xi_{1}^{\prime}(\alpha(t)) \in T_{\alpha(t)}^{\prime}$ for all $t$. Now, applying the isomorphism $\left(\pi_{*}\right)_{w}$ we have

$$
\left(\pi_{*}\right)_{w}(\vec{\alpha}(0))=\vec{\beta}(0)=Y
$$

and

$$
\left(\pi_{*}\right)_{w}\left(\nabla_{\vec{\alpha}(0)}^{\prime} \xi_{1}^{\prime}\right)=\tilde{\nabla}_{Y} \xi_{1} .
$$

But $\tilde{\nabla}_{Y} \xi_{1}=-A_{\xi_{1}} Y+\nabla_{\bar{Y}} \xi_{1}$, and since $\xi_{1}(x)=\xi$ and $\nabla_{Y}^{\frac{1}{Y}} \xi_{1}=0$, we have

$$
\tilde{\nabla}_{Y} \xi_{1}=-A_{\xi} Y \text {. }
$$

Thus, using (5), (6), (7) and applying $\left(\pi_{*}\right)_{w}$ to (3) we have

$$
\left(\pi_{*}\right)_{w} \vec{\eta}(0)=\cos r Y-\sin r A_{\xi} Y .
$$

Since $\vec{\eta}(0) \in T_{w}^{\prime}$, we know $\left(\pi_{*}\right)_{w} \vec{\eta}(0)=0$ if and only if $\vec{\eta}(0)=0$. From (8) we see that $\vec{\eta}(0)=0$ if and only if $k=\cot r$ is an eigen-value of $A_{\xi}$ and $Y$ is an eigen-vector of $k$. From (1) and (4) we see that this also gives necessary and sufficient conditions under which $F_{*}(X)=0$. If $\cot r$ is an eigen-value of multiplicity $\nu$, then it is clear that $F_{*}$ vanishes on a $\nu$-dimensional subspace of $T_{(x, r \xi)} N\left(M^{n}\right)$, i.e. $F_{*}$ has nullity $\nu$.

Q.E.D.

Since the degeneracies of $F_{*}$ of type (i) in Proposition 1 depend only on $r= \pm \pi / 2$ and not on $M^{n}$ or the point $x \in M^{n}$, they provide no information about $M^{n}$ itself. Thus such degeneracies will not be included in the following definition of a focal point of $\left(M^{n}, x\right)$. In the definition 
it is understood, as above, that $\xi$ is a unit vector in $T_{x}^{\perp}\left(M^{n}\right)$ and $-\pi / 2 \leq r \leq \pi / 2$.

Definition. A point $p \in P^{n+p}(C)$ is called a focal point of $\left(M^{n}, x\right)$ of multiplicity $\nu$ if $p=F(x, r \xi)$ and $\cot r$ is an eigen-value of multiplicity $\nu>0$ of $A_{\xi}$. (We say $p$ is a focal point of $M^{n}$ if $p$ is a focal point of $\left(M^{n}, x\right)$ for some $x \in M^{n}$.)

We now proceed to define the functions $L_{p}$. For $p, q \in P^{n+p}(C)$, and $z, w \in S^{2(n+p)+1}$ such that $\pi(z)=p, \pi(w)=q$, we define

$$
L_{p}(q)=\cos ^{-1}\left(|(z, w)|^{2}\right),
$$

where $0 \leq \cos ^{-1}(\quad) \leq \pi / 2$. One easily checks that the definition of $L_{p}(q)$ is independent of the choice of $z, w$.

We remark that $L_{p}(q)$ is essentially $d(p, q)$ the distance in $P^{n+p}(C)$ from $p$ to $q$ which is given by

$$
d(p, q)=\cos ^{-1}(|(z, w)|) .
$$

We use $L_{p}(q)$ rather than $d(p, q)$ to gain differentiability at points $q$ such that $L_{p}(q)=\pi / 2$. i.e. $(z, w)=0$.

For $p \in P^{n+p}(C), x \in M^{n}$, we define $L_{p}(x)=L_{p}(f(x))$. If $p \notin f\left(M^{n}\right)$, then the restriction of $L_{p}$ to $M^{n}$ is a differentiable function on $M^{n}$. From this point on, we will only consider $L_{p}$ such that $p \notin f\left(M^{n}\right)$. For such a point $p$, the following proposition describes the critical points of the function $L_{p}$ on $M^{n}$.

Proposition 2. Let $p \in P^{n+p}(C)$, and $x_{0} \in M^{n}$ such that $f\left(x_{0}\right) \neq p$. Then $x_{0}$ may be a critical point of $L_{p}$ in precisely the following 2 ways.

(i) If $L_{p}\left(x_{0}\right)=\pi / 2$, then $L_{p}$ has a degenerate maximum at $x_{0}$.

(ii) If $L_{p}\left(x_{0}\right)<\pi / 2, L_{p}$ has a critical point at $x_{0}$ if and only if $p$ can be expressed as $F\left(x_{0}, r \xi\right)$ where $\xi$ is a unit vector in $T_{x_{0}}^{\perp}\left(M^{n}\right)$ and $0<r<\pi / 2$. In this case,

(a) $x_{0}$ is a degenerate critical point if and only if $\cot r$ is an eigenvalue of $A_{\xi}$.

(b) The index of $L_{p}$ at a non-degenerate critical point $x_{0}$ equals the number of eigen-values, $k_{i}$, of $A_{\xi}$ such that $k_{i}>\cot r$. Each $k_{i}$ is counted with its multiplicity.

Proof. Fix $x_{0} \in M^{n}$, and let $p \in P^{n+p}(C)$. Fix $z_{0} \in S^{2(n+p)+1}$ such that 
$\pi\left(z_{0}\right)=p$. Let $X$ be a vector field on $M^{n}$, and let $X^{\prime}$ be the horizontal lift of $X$. For $x \in M^{n}$ and $w \in S^{2(n+p)+1}$ such that $\pi(w)=x$, we have

$$
\begin{aligned}
X L_{p}(x) & =\left(\pi_{*} X^{\prime}\right) L_{p}(x)=X^{\prime}\left(L_{p} \circ \pi\right)(w) \\
& =X^{\prime}\left(\cos ^{-1}\left(\left|\left(z_{0}, w\right)\right|^{2}\right)\right)=X^{\prime}\left(\cos ^{-1}\left(g\left(z_{0}, w\right)^{2}+g\left(z_{0}, i w\right)^{2}\right)\right) \\
& =\frac{-\left[2 g\left(z_{0}, w\right) X^{\prime}\left(g\left(z_{0}, w\right)\right)+2 g\left(z_{0}, i w\right) X^{\prime}\left(g\left(z_{0}, i w\right)\right)\right]}{\left(1-\left[g\left(z_{0}, w\right)^{2}+g\left(z_{0}, i w\right)^{2}\right]^{2}\right)^{1 / 2}} .
\end{aligned}
$$

But $X^{\prime}\left(g\left(z_{0}, w\right)\right)=g\left(z_{0}, X_{w}^{\prime}\right)$, and we obtain

$$
X L_{p}(x)=\frac{-2\left[g\left(z_{0}, w\right) g\left(z_{0}, X_{w}^{\prime}\right)+g\left(z_{0}, i w\right) g\left(z_{0}, i X_{w}^{\prime}\right)\right]}{\left(1-\left[g\left(z_{0}, w\right)^{2}+g\left(z_{0}, i w\right)^{2}\right]^{2}\right)^{1 / 2}} .
$$

In particular, to find $X L_{p}\left(x_{0}\right)$, we can choose $w_{0} \in S^{2(n+p)+1}$ such that $\pi\left(w_{0}\right)=x_{0}$, and such that $g\left(z_{0}, i w_{0}\right)=0$ and $0 \leq g\left(z_{0}, w_{0}\right)<1$. We know $g\left(z_{0}, w_{0}\right)<1$ since $p \neq f\left(x_{0}\right)$. From (9) we then obtain,

$$
X L_{p}\left(x_{0}\right)=\frac{-2\left[g\left(z_{0}, w_{0}\right) g\left(z_{0}, X_{w_{0}}^{\prime}\right)\right]}{\left(1-g\left(z_{0}, w_{0}\right)^{4}\right)^{1 / 2}} .
$$

From (10) we see that to have $X L_{p}\left(x_{0}\right)=0$, we must have either,

(i) $g\left(z_{0}, w_{0}\right)=0$ or

(ii) $g\left(z_{0}, X_{w_{0}}^{\prime}\right)=0$.

In case (i) $x_{0}$ is obviously a maximum of $L_{p}$ since $L_{p}\left(x_{0}\right)=\pi / 2$ which is the maximum value $L_{p}$ attains on $P^{n+p}(C)$. A direct calculation of the Hessian of $L_{p}$ at $x_{0}$ would show that the Hessian is degenerate, and hence $x_{0}$ is a degenerate maximum of $L_{p}$. We omit that argument here and appeal instead to the following geometric argument. The set of points

$$
P^{n+p-1}(C)=\left\{q \in P^{n+p}(C) \mid L_{p}(q)=\pi / 2\right\}
$$

is a totally geodesic hypersurface of $P^{n+p}(C)$ given by the image under the projection $\pi$ of $S^{2(n+p)-1}$ where

$$
S^{2(n+p)-1}=S^{2(n+p)+1} \cap\left\{w \in C^{n+p+1} \mid\left(z_{0}, w\right)=0\right\} .
$$

This $P^{n+p-1}(C)$ is the set of zeroes of an analytic function on $P^{n+p}(C)$. If $f\left(x_{0}\right) \in f\left(M^{n}\right) \cap P^{n+p-1}$, then in a neighborhood $U$ of $x_{0}$ in $M^{n}$, the set $f(U) \cap P^{n+p-1}$ is the set of zeroes of an analytic function on $U$. It follows essentially from the Weierstrass Preparation Theorem (see [1], p. 37-43) that $f(U) \cap P^{n+p-1}(C)$ is a sub-variety of $U$ of dimension $j$, 
where $j \geq n-1$. For $n \geq 2$, this illustrates that $x_{0}$ is not an isolated maximum of $L_{p}$ on $M^{n}$; clearly then, $x_{0}$ is a degenerate maximum. This proves (i).

Now we assume $g\left(z_{0}, w_{0}\right)>0$, i.e. $L_{p}\left(x_{0}\right)<\pi / 2$. Since $L_{p}\left(x_{0}\right) \neq 0$, we know $g\left(z_{0}, w_{0}\right)<1$; and so there exists $r, 0<r<\pi / 2$, so that $\cos r=$ $g\left(z_{0}, w_{0}\right)$. Then it is easy to show,

$$
z_{0}=\cos r w_{0}+\sin r \xi^{\prime}
$$

where $\xi^{\prime} \in T_{w_{0}}^{\prime}$ and $\left\|\xi^{\prime}\right\|=1$. Then,

$$
g\left(z_{0}, X_{w_{0}}^{\prime}\right)=\sin r g\left(\xi^{\prime}, X_{w_{0}}^{\prime}\right)
$$

for $X_{w_{0}}^{\prime}$ the horizontal lift of $X \in T_{x_{0}}\left(M^{n}\right)$. This and (10) imply that if $L_{p}\left(x_{0}\right)<\pi / 2$, then $x_{0}$ is a critical point of $L_{p}$ if and only if $\pi_{*}\left(\xi^{\prime}\right)=$ $\xi \in T_{x_{0}}^{\perp}\left(M^{n}\right)$; in that case, $p=F\left(x_{0}, r \xi\right)$ and we have proven (ii).

Now for $p=F\left(x_{0}, r \xi\right), 0<r<\pi / 2$, we wish to prove (a) and (b). We first compute the Hessian of $L_{p}$ at $x_{0}$. Let $X, Y$ be vector fields on $M^{n}$ and $X^{\prime}, Y^{\prime}$ their respective horizontal lifts. We have shown

$$
X L_{p}(x)=\frac{-2\left[g\left(z_{0}, w\right) g\left(z_{0}, X_{w}^{\prime}\right)+g\left(z_{0}, i w\right) g\left(z_{0}, i X_{w}^{\prime}\right)\right]}{\left(1-\left[g\left(z_{0}, w\right)^{2}+g\left(z_{0}, i w\right)^{2}\right]^{2}\right)^{1 / 2}}
$$

where $\pi(w)=x$.

We now find $Y X L_{p}\left(x_{0}\right)$. For $w_{0}$ as chosen above,

$$
g\left(z_{0}, X_{w_{0}}^{\prime}\right)=g\left(z_{0}, i X_{w_{0}}^{\prime}\right)=0 \text { and } g\left(z_{0}, i w_{0}\right)=0 .
$$

We also know that

$$
Y^{\prime}\left(g\left(z_{0}, X_{w}^{\prime}\right)\right)=g\left(z_{0}, D_{Y^{\prime}} X^{\prime}\right)
$$

where $D$ is the Euclidean covariant derivative in $C^{n+p+1}$. Using these facts we differentiate (9) to find $Y X L_{p}(x)$ and then evaluate at $x_{0}$ obtaining

$$
Y X L_{p}\left(x_{0}\right)=\frac{-\left.2 g\left(z_{0}, w_{0}\right) g\left(z_{0}, D_{Y^{\prime}} X^{\prime}\right)\right|_{w_{0}}}{\left(1-g\left(z_{0}, w_{0}\right)^{4}\right)^{1 / 2}}
$$

But we know $g\left(z_{0}, w_{0}\right)=\cos r$ so

$$
1-g\left(z_{0}, w_{0}\right)^{4}=1-\cos ^{4} r=\sin ^{2} r\left(1+\cos ^{2} r\right),
$$

and we re-write (12) as 


$$
Y X L_{p}\left(x_{0}\right)=\frac{-\left.2 \cos r g\left(z_{0}, D_{Y^{\prime}} X^{\prime}\right)\right|_{w_{0}}}{\left(1+\cos ^{2} r\right)^{1 / 2} \sin r}
$$

From well-known properties of the embedding of $S^{2(n+p)+1}$ in $C^{n+p+1}$, we know that for any $w \in S^{2(n+p)+1}$,

$$
\left.D_{Y^{\prime}} X^{\prime}\right|_{w}=\left.\nabla_{Y^{\prime}}^{\prime} X^{\prime}\right|_{w}-g\left(X^{\prime}, Y^{\prime}\right) w
$$

We can also write

$$
\nabla_{Y^{\prime}}^{\prime}, X^{\prime}=W+\alpha^{\prime}\left(X^{\prime}, Y^{\prime}\right)
$$

where $\pi_{*}(W)=\nabla_{Y} X$, where $\nabla$ is the covariant derivative on $M^{n}$, and

$$
\pi_{*}\left(\alpha^{\prime}\left(X^{\prime}, Y^{\prime}\right)\right)=\alpha(X, Y),
$$

where $\alpha(X, Y)$ is the second fundamental form of the immersion $f$. Now since $\pi_{*}\left(\xi^{\prime}\right) \in T \frac{\perp}{x_{0}}\left(M^{n}\right)$, we have $g\left(\xi^{\prime}, W\right)=0$. Since $\xi^{\prime}, W \in T_{w_{0}}^{\prime}$, we know

$$
g\left(w_{0}, \xi^{\prime}\right)=0=g\left(w_{0}, W\right) .
$$

Thus (11), (14), and (15) yield,

$$
\left.g\left(z_{0}, D_{Y^{\prime}} X^{\prime}\right)\right|_{w_{0}}=\left.\sin r g\left(\xi^{\prime}, \alpha^{\prime}\left(X^{\prime}, Y^{\prime}\right)\right)\right|_{w_{0}}-\left.\cos r g\left(X^{\prime}, Y^{\prime}\right)\right|_{w_{0}} .
$$

But

$$
\begin{aligned}
\left.g\left(\xi^{\prime}, \alpha^{\prime}\left(X^{\prime}, Y^{\prime}\right)\right)\right|_{w_{0}} & =\left.\tilde{g}(\xi, \alpha(X, Y))\right|_{x_{0}} \\
& =\left.\tilde{g}\left(A_{\xi} X, Y\right)\right|_{x_{0}} .
\end{aligned}
$$

Thus (16) becomes

$$
\left.g\left(z_{0}, D_{Y^{\prime}} X^{\prime}\right)\right|_{w_{0}}=\sin r \tilde{g}\left(A_{\xi} X, Y\right)-\left.\cos r \tilde{g}(X, Y)\right|_{x_{0}}
$$

and (13) becomes

$$
Y X L_{p}\left(x_{0}\right)=\left.\frac{2 \cos r}{\left(1+\cos ^{2} r\right)^{1 / 2}} \tilde{g}\left(\left(-A_{\xi}+\cot r I\right) X, Y\right)\right|_{x_{0}}
$$

where $I$ is the identity endomorphism on $T_{x_{0}}\left(M^{n}\right)$.

From this expression for the terms of the Hessian of $L_{p}$ at $x_{0}$, we conclude that $x_{0}$ is a degenerate critical point of $L_{p}$, if and only if $\cot r$ $=k$ for $k$ an eigen-value of $A_{\xi}$. This proves (a).

The index of $L_{p}$ at a non-degenerate critical point $x_{0}$ is defined to be the number of negative eigen-values of the Hessian of $L_{p}$ at $x_{0}$. For $\cot r \neq k_{i}$ for any eigen-value $k_{i}$ of $A_{\xi}$, we see from (17) that the index 
of $L_{p}$ at $x_{0}$ is the number of $k_{i}$ such that $k_{i}>\cot r$. This proves (b). Q.E.D.

Propositions (1) and (2) yield immediately the following theorem:

THEOREM 1 (Index Theorem for $L_{p}$ ). Let $p=F(x, r \xi)$ for $0<r<\pi / 2$. Suppose $L_{p}$ has a non-degenerate critical point at $x$. Then the index of $L_{p}$ at $x$ equals the number of focal points of $\left(M^{n}, x\right)$ which lie on the geodesic in $P^{n+p}(C)$ from $f(x)$ to $p$. Each focal point is counted with its multiplicity.

\section{Section 3-A Characterization of $P^{n}(C)$ and $Q^{n}(C)$.}

We now proceed to the main result of this article which we state here.

THEOREM 2. Let $M^{n}(n \geq 2)$ be a connected, complete, complex $n$ dimensional Kählerian manifold which is holomorphically and isometrically immersed in $P^{n+p}(C)$. If there exists a dense subset $D$ of $P^{n+p}(C)$ such that every function of the form $L_{p}, p \in D$, has index 0 or $n$ at any of its non-degenerate critical points, then $M^{n}$ is embedded in $P^{n+p}(C)$ as $P^{n}(C)$ or $Q^{n}(C)$.

In the above statement, $P^{n}(C)$ stands for a totally geodesic submanifold of $P^{n+p}(C)$, and $Q^{n}(C)$ is the standard complex quadric hypersurface of some totally geodesic $P^{n+1}(C)$. In $P^{n+1}(C)$ has homogeneous co-ordinates $\left(z_{0}, \cdots, z_{n+1}\right)$, then $Q^{n}(C)$ is defined by the equation

$$
z_{0}^{2}+\cdots+z_{n+1}^{2}=0 .
$$

In the remainder of this section we assume that $M^{n}$ satisfies the hypotheses of Theorem 2. To begin the proof of Theorem 2, we state the following proposition. Its proof, which we omit here, depends on Propositions 1 and 2. With minor changes, the proof is identical to the corresponding proposition for submanifolds of $\boldsymbol{R}^{m}$ proven by Nomizu and Rodriguez ([6], p. 199).

Proposition 3. Let $D$ be a dense subset of $P^{n+p}(C)$. Assume that for $p \in P^{n+p}(C), L_{p}$ has a non-degenerate critical point of index $j$ at $x \in M^{n}$. Then there exists $q \in D, y \in M^{n}$ such that $L_{q}$ has a non-degenerate critical point of index $j$ at $y$ ( $q$ and $y$ may be chosen as close to $p$ and $x$, respectively, as desired). 
Using Proposition 3 and the Index Theorem, we now prove the following proposition which is sufficient to complete the proof of Theorem 2 for the case of co-dimension $p=1$.

Proposition 4. Let $x \in M^{n}$ and $\xi$ be a unit-length vector in $T_{\frac{1}{x}}^{\perp}\left(M^{n}\right)$. Then there exists $\lambda \geq 0$ such that $A_{\xi}^{2}=\lambda^{2} I$ on $T_{x}\left(M^{n}\right)$.

Proof. Fix $x \in M^{n}$ and $\xi$ a unit-length vector in $T \frac{1}{x}\left(M^{n}\right)$. If $A_{\xi}$ has no non-zero eigen-values, then $A_{\xi}=0$ and the proof is complete.

Suppose $A_{\xi}$ has at least one non-zero eigen-value. It is known that $A_{\xi}$ must have the form

$$
A_{\xi}=\left[\begin{array}{llllllll}
k_{1} & & & & & & & \\
& k_{2} & & & & & & \\
& & \cdot & & & & & \\
& & & k_{n} & & & & \\
& & & & -k_{1} & & & \\
& & & & & -k_{2} & & \\
& & & & & & \ddots & \ddots
\end{array}\right]
$$

when diagonalized for $k_{i} \geq 0,1 \leq i \leq n$. Let $\lambda$ be the largest of the eigen-values. If $k_{i}=\lambda$ for $1 \leq i \leq n$, then $A_{\xi}^{2}=\lambda^{2} I$ and the proof is finished. If $k_{i} \neq \lambda$ for some $i$, let $\beta \geq 0$ be the second largest of the non-negative eigen-values. Choose $r, 0<r<\pi / 2$, such that $\beta<\cot r<\lambda$. For $p=F(x, r \xi)$, Proposition 2 implies that $L_{p}$ has a non-degenerate critical point of index $j$ at $x$ where $0<j \leq 2 n$. Since $\lambda>\cot r>k_{i}$, for any $k_{i} \neq \lambda$, Proposition 2 also implies that $j$ equals the multiplicity of $\lambda$.

For $D$ as in Theorem 2, Proposition 3 implies that there exists $q \in D$ and $y \in M^{n}$ such that $L_{q}$ has a non-degenerate critical point of index $j$ at $y$. Since $j>0$, the hypothesis on the index of $L_{q}, q \in D$, at a nondegenerate critical point implies that $j=n$. Thus $\lambda$ has multiplicity equal to $n$, and again we conclude $A_{\xi}^{2}=\lambda^{2} I$. Q.D.

Remark 1. For the case when $M^{n}$ is a hypersurface of $P^{n+1}(C)$, Proposition 4 yields the proof of Theorem 2 in the following way.

The condition that $A_{\xi}^{2}=\lambda^{2} I$ for any $\xi \in T \frac{\perp}{x}\left(M^{n}\right)$ and any $x \in M^{n}$ implies that $M^{n}$ is an Einstein manifold. This is clear from the following 
equation (see [8], p. 253). For $S(X, Y)$, the Ricci tensor of $M^{n}$, it is true that

$$
\begin{aligned}
S(X, Y) & =-2 \tilde{g}\left(A_{\xi}^{2} X, Y\right)+2(n+1) \tilde{g}(X, Y) \\
& =2\left(n+1-\lambda^{2}\right) \tilde{g}(X, Y) .
\end{aligned}
$$

Since the real dimension of $M^{n}$ exceeds 2, a classical theorem (see [4], Vol. I, p. 292) implies that $2\left(n+1-\lambda^{2}\right)$ is indeed constant on $M^{n}$. Thus $M^{n}$ is an Einstein manifold. Theorem 2 then follows from the following result of Brian Smyth ([8], p. 265).

TheOREM (Smyth). For $n \geq 2, P^{n}(C)$ and $Q^{n}(C)$ are the only complex hypersurfaces of $P^{n+1}(C)$ which are complete and Einstein.

(end of Remark 1).

\section{Section 4-Reducing the co-dimension.}

To complete the proof of Theorem 2 for arbitrary co-dimensions, we will show that under the hypotheses of Theorem $2, M^{n}$ is actually a hypersurface of a totally geodesic $P^{n+1}(C) \subset P^{n+p}(C)$.

We first must introduce the concept of the first normal space of $M^{n}$ at $x \in M^{n}$.

DEFINITION. For $x \in M^{n}$, the first normal space, $N_{1}(x)$, is the orthogonal complement in $T \frac{\perp}{x}\left(M^{n}\right)$ of the set

$$
N_{0}(x)=\left\{\xi \in T_{x}^{\perp}\left(M^{n}\right) \mid A_{\xi}=0\right\} .
$$

We define a new inner product, $\langle$,$\rangle , on N_{1}(x)$ by

$$
\langle\xi, \eta\rangle=\operatorname{trace} A_{\xi} A_{\eta} \quad \text { for } \xi, \eta \in N_{1}(x) \text {. }
$$

One easily checks that $\langle$,$\rangle is a positive definite inner product on N_{1}(x)$, and that for $\xi, \eta \in N_{1}(x)$,

$$
\langle J \xi, J \eta\rangle=\langle\xi, \eta\rangle
$$

and

$$
\langle\xi, J \xi\rangle=0 \text {. }
$$

For $\xi \in N_{1}(x)$, Proposition 4 implies $A_{\xi}^{2}=\lambda^{2} I$ for $\lambda>0$. Then it is easy to see that $T_{x}\left(M^{n}\right)$ can be decomposed as

$$
T_{x}\left(M^{n}\right)=T_{\xi}^{+} \oplus T_{\xi}^{-}
$$


where

$$
T_{\xi}^{+}=\left\{X \in T_{x}\left(M^{n}\right) \mid A_{\xi} X=\lambda X\right\}
$$

and

$$
T_{\xi}^{-}=\left\{X \in T_{x}\left(M^{n}\right) \mid A_{\xi} X=-\lambda X\right\} .
$$

It is a simple matter to show that if $X \in T_{\xi}^{+}$, then $J X \in T_{\xi}^{-}$; and if $X \in T_{\xi}^{-}$, then $J X \in T_{\xi}^{+}$. We employ the inner product $\langle$,$\rangle in the follow-$ ing proposition to prove that $N_{1}(x)$ has complex dimension no larger than 1 for all $x \in M^{n}$.

Proposition 5. Let $x \in M^{n}$ and let $k$ be the complex dimension of $N_{1}(x)$. Then $k \leq 1$.

Proof. Assume $k>1$. Choose $\xi_{1}, \cdots, \xi_{k}$ so that with respect to the inner product $\langle$,$\rangle , the vectors \xi_{1}, \cdots, \xi_{k}, J \xi_{1}, \cdots, J \xi_{k}$ from an orthonormal basis for $N_{1}(x)$.

We know there is a positive function $\lambda$ on $N_{1}(x)$ such that $A_{\xi}^{2}=\lambda^{2}(\xi) I$ for any $\xi \in N_{1}(x)$. If $e_{1}, \cdots, e_{n}$ are an orthonormal basis for $T^{+}=T_{\xi_{1}}^{+}$, then $J e_{1}, \cdots, J e_{n}$ are an orthonormal basis for $T^{-}=T_{\xi_{1}}^{-}$. With respect to the basis $\Omega$ for $T_{x}\left(M^{n}\right)$,

$$
\Omega=\left\{e_{1}, \cdots, e_{n}, J e_{1}, \cdots, J e_{n}\right\},
$$

the endomorphism $A_{\xi_{1}}$ is represented by the matrix

$$
A_{\xi_{1}}=\left[\begin{array}{cc}
\lambda\left(\xi_{1}\right) I_{n} & 0 \\
0 & -\lambda\left(\xi_{1}\right) I_{n}
\end{array}\right]
$$

where $I_{n}$ is an $n \times n$ identity matrix.

Fix $j, 2 \leq j \leq k$. Consider $X \in T^{+}$, and suppose $A_{\xi}, X=Y+Z$ where $Y \in T^{+}, Z \in T^{-}$. First of all, we have

$$
A_{\xi_{1}+\xi_{j}}^{2} X=\lambda^{2}\left(\xi_{1}+\xi_{j}\right) X .
$$

But also we find,

$$
\begin{aligned}
A_{\xi_{1}+\xi_{j}}^{2} X & =A_{\xi_{1}+\xi_{j}} A_{\xi_{1}+\xi_{j}} X=A_{\xi_{1}}^{2} X+\left(A_{\xi_{1}} A_{\xi_{j}}+A_{\xi_{j}} A_{\xi_{1}}\right) X+A_{\xi_{j}}^{2} X \\
& =\lambda^{2}\left(\xi_{1}\right) X+\lambda^{2}\left(\xi_{j}\right) X+\lambda\left(\xi_{1}\right)(Y-Z)+\lambda\left(\xi_{1}\right)(Y+Z) \\
& =\left(\lambda^{2}\left(\xi_{1}\right)+\lambda^{2}\left(\xi_{j}\right)\right) X+2 \lambda\left(\xi_{1}\right) Y
\end{aligned}
$$

Then (21) and (22) yield 


$$
Y=\mu X, \quad \text { where } \mu=\left[\lambda^{2}\left(\xi_{1}+\xi_{j}\right)-\lambda^{2}\left(\xi_{1}\right)-\lambda^{2}\left(\xi_{j}\right)\right] / 2 \lambda\left(\xi_{1}\right) .
$$

Since we see that $\mu$ does not depend on the choice of $X$, we have shown that for any $X \in T^{+}$,

$$
A_{\varepsilon}, X=\mu X+Z \quad \text { where } Z \in T^{-} \text {. }
$$

From (24) we can also compute for $X \in T^{+}$,

$$
A_{\xi j} J X=-J A_{\xi j} X=-J(\mu X+Z)=-\mu J X-J Z .
$$

Equations (24) and (25) and the fact that $A_{\xi,}$ is symmetric imply that with respect to the basis $\Omega, A_{\xi j}$ has the form

$$
A_{\xi_{j}}=\left[\begin{array}{cc}
\mu I_{n} & { }^{t} B \\
B & -\mu I_{n}
\end{array}\right]
$$

where $B$ is an $n \times n$ matrix.

Since $\xi_{1}$ and $\xi_{j}$ are orthogonal with respect to $\langle$,$\rangle , we know$

$$
\operatorname{trace} A_{\xi_{1}} A_{\xi_{j}}=0 \text {. }
$$

However, equations (20) and (26) imply that with respect to the basis $\Omega$,

$$
A_{\xi_{1}} A_{\xi_{j}}=\left[\begin{array}{cc}
\lambda\left(\xi_{1}\right) \mu I_{n} & { }^{t} B \\
B & \lambda\left(\xi_{1}\right) \mu I_{n}
\end{array}\right] \text {. }
$$

From (28) we compute trace $A_{\xi_{1}} A_{\xi_{j}}=2 n \lambda\left(\xi_{1}\right) \mu$. Comparing this with (27), we conclude $\mu=0$, since $\lambda\left(\xi_{1}\right)>0$. Hence (26) becomes

$$
A_{\xi_{j}}=\left[\begin{array}{rr}
0 & { }^{t} B \\
B & 0
\end{array}\right] \text {. }
$$

From the fact that $\tilde{\nabla}$ is a Kählerian connection, one easily shows that $A_{J \xi_{j}}=J A_{\xi_{j}}$. From (29), we see that as a matrix,

$$
A_{J_{j}}=J A_{\xi_{j}}=\left[\begin{array}{cc}
0 & -I_{n} \\
I_{n} & 0
\end{array}\right]\left[\begin{array}{cc}
0 & { }^{t} B \\
B & 0
\end{array}\right]=\left[\begin{array}{cc}
-B & 0 \\
0 & { }^{t} B
\end{array}\right] \text {. }
$$

This shows that $A_{J \xi}$ maps $T^{+}$into $T^{+}$and $T^{-}$into $T^{-}$. This fact and computations similar to those leading to (23) show that for $X \in T^{+}$,

$$
A_{J \xi j} X=\nu X,
$$

where

$$
\nu=\left[\lambda^{2}\left(\xi_{1}+J \xi_{j}\right)-\lambda^{2}\left(\xi_{1}\right)-\lambda^{2}\left(J \xi_{j}\right)\right] / 2 \lambda\left(\xi_{1}\right)
$$


Thus we can represent $A_{J \xi}$ as,

$$
A_{J \xi_{j}}=\left[\begin{array}{cc}
\nu I_{n} & 0 \\
0 & -\nu I_{n}
\end{array}\right]
$$

Now equations (20) and (30) imply that $A_{\xi_{1}} A_{J \xi_{j}}=\lambda\left(\xi_{1}\right) \nu I$ on $T_{x}\left(M^{n}\right)$, and

$$
\operatorname{trace} A_{\xi_{1}} A_{J \xi_{j}}=2 n \lambda\left(\xi_{1}\right) \nu \text {. }
$$

But $\left\langle\xi_{1}, J \xi_{j}\right\rangle=0$, and so trace $A_{\xi_{1}} A_{J \xi_{j}}=0$. Comparing this with (31), we conclude $\nu=0$. Then (30) implies $A_{J \xi_{j}}=0$ which implies $A_{\xi_{j}}$ $=0$, and $\xi_{j} \notin N_{1}(x)$. This is true for $2 \leq j \leq k$, and we have obtained a contradiction if we assume $k>1$. Thus, $k \leq 1$.

Q.E.D.

We first want to make it clear that we have no further use for the inner product $\langle$,$\rangle . Any subsequent references to metric properties such$ as orthogonality are made with respect to the metrics $g$ or $\tilde{g}$.

We now begin to reduce the co-dimension. The argument is similar to that used by Cartan to show that an umbilical submanifold of $\boldsymbol{R}^{m}$ which is not totally geodesic must be a Euclidean sphere embedded in $\boldsymbol{R}^{m}$ (see [2], p. 231).

Proposition 5 enables us to define a function $\lambda$ on $M^{n}$ in the following way. Let $\alpha(X, Y)$ be the second fundamental form of $M^{n}$ in $P^{n+p}(C)$. If $\alpha(X, Y)=0$ at $x \in M^{n}$, we set $\lambda(x)=0$. If $\alpha(X, Y) \neq 0$ at $x \in M^{n}$, then by Proposition 5, $N_{1}(x)$ has complex dimension 1 . We define $\lambda(x)$ to be the well-defined positive number such that $A_{\xi}^{2}=\lambda^{2}(x) I$ for any unit vector $\xi$ in $N_{1}(x)$. It is easy to show from the obvious dependence of $\lambda$ on $\alpha(X, Y)$ that $\lambda$ is continuous on $M^{n}$. We omit that proof here, however, and next prove the following.

Proposition 6. Let $x \in M^{n}$ and suppose the second fundamental form $\alpha(X, Y) \neq 0$ at $x$. Then there is a neighborhood $U$ of $x$ in $M^{n}$ on which the function $\lambda$ is constant.

Proof. Let $U$ be a neighborhood of $x$ on which $\alpha(X, Y) \neq 0$. Then by Proposition 5, $N_{1}(u)$ has constant dimension 1 on $U$. It is easy to show, then, that there exists a unit-length vector field $\xi_{1}$, on $U$ such that

$$
N_{1}(u)=\operatorname{span}\left\{\xi_{1}, J \xi_{1}\right\} \quad \text { for every } u \in U \text {. }
$$

Let $\xi_{2}, \cdots, \xi_{p}$ be unit-length normal vector fields on $U$ such that $\xi_{1}, \xi_{2}$, $\cdots, \xi_{p}, J \xi_{1}, \cdots, J \xi_{p}$ are an orthonormal basis for $T \frac{\perp}{u}\left(M^{n}\right)$ for any $u \in U$. 
Fix an arbitrary point $u \in U$. The following equation defines the the tensors $s_{k j}$ and $t_{k j}$ on $T_{u}\left(M^{n}\right)$,

$$
\nabla_{X}^{\frac{1}{X}} \xi_{j}=\sum_{k=1}^{p} s_{k j}(X) \xi_{k}+\sum_{k=1}^{p} t_{k j}(X) J \xi_{k} \quad \text { for } X \in T_{u}\left(M^{n}\right) .
$$

The fact that $\nabla^{\perp}$ is a Kählerian connection readily implies

$$
s_{k j}(X)=-s_{j k}(X)
$$

and

$$
t_{k j}(X)=t_{j k}(X) .
$$

Now we know $A_{\xi_{j}}=A_{J \xi_{j}}=0$ for $2 \leq j \leq p$. This fact and (33) imply that Codazzi's equation for $A_{\xi_{1}}$ reduces to

$$
\left(\nabla_{X} A_{\xi_{1}}\right)(Y)-t_{11}(X) J A_{\xi_{1}}(Y)=\left(\nabla_{Y} A_{\xi_{1}}\right)(X)-t_{11}(Y) J A_{\xi_{1}}(X) .
$$

Let $X, Y \in T^{+-}=T_{\xi_{1}}^{+}(u)$ such that $X, Y$ are linearly independent, and suppose

$$
\begin{array}{ll}
\nabla_{X} Y=X_{1}+X_{2} & \text { for } X_{1} \in T^{+}, X_{2} \in T^{-}, \\
\nabla_{Y} X=Y_{1}+Y_{2} & \text { for } Y_{1} \in T^{+}, Y_{2} \in T^{-}
\end{array}
$$

Using the above equations and recalling the following equations,

$$
\begin{array}{ll}
A_{\xi_{1}} Z=\lambda Z & \text { for } Z \in T^{+}, \\
A_{\xi_{1}} Z=-\lambda Z & \text { for } Z \in T^{-},
\end{array}
$$

we find after some calculation that (35) becomes

$$
(X \lambda) Y+2 \lambda X_{2}+t_{11}(X) \lambda J Y=(Y \lambda) X+2 \lambda Y_{2}+t_{11}(Y) \lambda J X .
$$

But $X_{2}, Y_{2}, J X, J Y$ are in $T^{-}$, and the component of (36) in $T^{+}$is,

$$
(X \lambda) Y=(Y \lambda) X \text {. }
$$

The linear independence of $X$ and $Y$ implies that $X \lambda=0$. This is true for any $X \in T^{+}$. A similar calculation shows $X \lambda=0$ for any $X \in T^{-}$. So we have $X \lambda=0$ for any $X \in T_{u}\left(M^{n}\right)$ for any $u \in U$. This is implies $\lambda$ is constant on $U$.

Q.E.D.

Proposition 6 enables us to prove that $N_{1}(x)$ has constant dimension on $M^{n}$ as follows.

Proposition 7. $N_{1}(x)$ has constant dimension on $M^{n}$. 
Proof. If the second fundamental form $\alpha(X, Y)=0$ for all $x \in M^{n}$, then $N_{1}(x)$ has constant dimension 0 ; and the proof is complete.

Suppose $\alpha(X, Y) \neq 0$ at $x_{0} \in M^{n}$. Consider the set $S$ defined by

$$
S=\left\{x \in M^{n} \mid \lambda(x)=\lambda\left(x_{0}\right)\right\} .
$$

Since $\lambda$ is continuous on $M^{n}$, we know $S$ is closed. However Proposition 6 implies $S$ is open. Since $x_{0} \in S$, we know $S \neq \phi$; so the connectedness of $M^{n}$ implies $S=M^{n}$. Hence $\lambda=\lambda\left(x_{0}\right)$ on $M^{n}$, and $N_{1}(x)$ has constant dimension 1 on $M^{n}$.

Q.E.D.

In the case where $N_{1}(x)$ has constant dimension $0, M^{n}$ is totally geodesic, and hence $M^{n}=P^{n}(C)$. To complete the proof of Theorem 2, we must show that when $N_{1}(x)$ has constant dimension 1 , we can reduce the co-dimension to 1 .

Let $U$ be any co-ordinate neighborhood of $M^{n}$. As before we choose orthonormal vector fields $\xi_{1}, \cdots, \xi_{p}$ so that $\xi_{1}, \cdots, \xi_{p}, J \xi_{1}, \cdots, J \xi_{p}$ span $T T_{u}^{\perp}\left(M^{n}\right)$ for any $u \in U$, and such that $\xi_{1}, J \xi_{1}$ span $N_{1}(u)$ for any $u \in U$. We then prove, .

Proposition 8. For any $x \in U$ and $X \in T_{x}\left(M^{n}\right)$ the following equations are true:

(i) $\nabla_{X}^{\frac{1}{X}} \xi_{1}=t_{11}(X) J \xi_{1}$

(ii) For $j \geq 2, \nabla_{\bar{X}}^{\frac{1}{X}} \xi_{j}$ and $\nabla_{\bar{X}} J \xi_{j} \in \operatorname{span}\left\{\xi_{k}, J \xi_{k} \mid 2 \leq k \leq p\right\}$, i.e. $N_{1}(x)$ and $N_{0}(x)$ are invariant with respect to $\nabla^{\perp}$.

Proof. For ease of notation, let $A_{j}=A_{\xi}, 1 \leq j \leq p$. For any fixed $j, 2 \leq j \leq p$, Codazzi's equation says the following,

$$
\left(\nabla_{X} A_{j}\right)(Y)-\sum_{k=1}^{p} s_{k j}(X) A_{k}(Y)-\sum_{k=1}^{p} t_{k j}(X) J A_{k}(Y)
$$

is symmetric in $X$ and $Y$.

Since $A_{j}=0$, then $\left(\nabla_{X} A_{j}\right)=0$ and Codazzi's equation can be written as :

$$
s_{1 j}(X) A_{1}(Y)+t_{1 j}(X) J A_{1}(Y)=s_{1 j}(Y) A_{1}(X)+t_{1 j}(Y) J A_{1}(X) .
$$

Choose $X, Y$ linearly independent vectors in $T_{\xi_{1}}^{+}(x)$; then since $A_{1}(X)$ $=\lambda X$ and $A_{1}(Y)=\lambda Y,(38)$ becomes

$$
s_{1 j}(X) \lambda Y+t_{1 j}(X) \lambda J Y=s_{1 j}(Y) \lambda X+t_{1 j}(Y) \lambda J X .
$$


But $X, Y, J X, J Y$ are linearly independent, so (39) implies

$$
s_{1 j}(X)=t_{1 j}(X)=0, \quad 2 \leq j \leq p .
$$

A similar calculation shows that (40) holds for $X \in T_{\xi_{1}}^{-}(x)$, and hence (40) holds for all $X \in T_{x}\left(M^{n}\right)$. We recall that for $1 \leq j \leq p$,

$$
\nabla_{\frac{1}{X} \xi_{j}}=\sum_{k=1}^{p} s_{k j}(X) \xi_{j}+\sum_{k=1}^{p} t_{k j}(X) J \xi_{k} .
$$

Then $s_{k j}=-s_{j k}$ and $t_{k j}=t_{j k}$ and (40) imply that for $j=1$, (32) becomes

$$
\nabla_{\bar{X}} \frac{\perp}{\xi_{1}}=t_{11}(X) J \xi_{1}
$$

proving (i). For the same reasons, for $j>1$, (32) becomes

$$
\nabla_{\frac{1}{X}} \xi_{j}=\sum_{k=2}^{p} s_{k j}(X) \xi_{k}+\sum_{k=2}^{p} t_{k j}(X) J \xi_{k} .
$$

Then $\nabla_{\bar{X}}^{\frac{1}{X}} J \xi_{j}=J\left(\nabla_{\bar{X}}^{\frac{1}{X}} \xi_{j}\right)$ and (42) prove (ii).

Q.E.D.

Finally Proposition 8 and the fact that $N_{1}(x)$ has constant complex dimension 1 will imply that $f\left(M^{n}\right) \subset P^{n+1}(C)$ after we prove the following proposition. We note that J. Erbacher, [3], has proven a corresponding result for real submanifolds of real space forms. With minor changes, the following proposition can be proven for submanifolds of $\boldsymbol{C}^{n+p}$ and the complex hyperbolic space form, $H^{n+p}(\boldsymbol{C})$.

Proposition 9. Let $f: M^{n} \rightarrow P^{n+p}(C)$ be a holomorphic and isometric immersion of a connected, complete, complex n-dimensional Kählerian manifold $M^{n}$ into $P^{n+p}(C)$. Suppose the first normal space $N_{1}(x)$ has constant dimension $k$, and is parallel with respect to the normal connection. Then there is a totally geodesic $(n+k)$-dimensional submanifold, $P^{n+k}(C)$, such that $f\left(M^{n}\right) \subset P^{n+k}(C)$.

Proof. We first remark that since $N_{1}(x)$ is parallel with respect to $\nabla^{\perp}$, so is its complement $N_{0}(x)$. Let $U$ be a co-ordinate neighborhood of $M^{n}$ and fix $x_{0} \in U$.

Choose $\xi_{1}, \cdots, \xi_{p} \in T_{x_{0}}^{\perp}\left(M^{n}\right)$ so that the following equations hold for $x=x_{0}$,

$$
N_{1}(x)=\operatorname{span}\left\{\xi_{j}, J \xi_{j} \mid 1 \leq j \leq k\right\}
$$

and 


$$
N_{0}(x)=\operatorname{span}\left\{\xi_{j}, J \xi_{j} \mid k+1 \leq j \leq p\right\} .
$$

Extend $\xi_{1}, \cdots, \xi_{p}$ to vector fields on $U$ by parallel translation with respect to $\nabla^{\perp}$ along geodesics of $M^{n}$. Then (43) and (44) hold for any $x \in U$.

Let $\xi_{j}^{\prime}$ denote the horizontal lift to $T_{w}^{\prime}$ of $\xi_{j}(\pi(w))$ where $\pi(w) \in U$. Fix $w_{0} \in S^{2(n+p)+1}$ so that $\pi\left(w_{0}\right)=x_{0}$. Let $V_{w_{0}}$ be the real affine subspace of $\boldsymbol{C}^{n+p+1}$ through $w_{0}$ given by

$$
V_{w_{0}}=\operatorname{span}\left\{\xi_{j}^{\prime}\left(w_{0}\right), i \xi_{j}^{\prime}\left(w_{0}\right) \mid k+1 \leq j \leq p\right\} .
$$

Let $W_{w_{0}}$ be the real affine space through $w_{0}$ of real dimension $2(n+k+1)$ which is orthogonal to $V_{w_{0}}$. Since the vector $-w_{0} \in W_{w_{0}}$, we know that the affine space $W_{w_{0}}$ passes through the origin in $C^{n+p+1}$. Hence the set

$$
S^{2(n+k)+1} \equiv W_{w_{0}} \cap S^{2(n+p)+1}
$$

is a great $(2(n+k)+1)$-dimensional sphere in $S^{2(n+p)+1}$. The set $P^{n+k}(C)$ $=\pi\left(S^{2(n+k)+1}\right)$ is an $(n+k)$-dimensional totally geodesic submanifold of $P^{n+p}(C)$. We will show that $f\left(M^{n}\right) \subset P^{n+k}(C)$.

We first prove $f(U) \subset P^{n+k}(C)$. Fix $u \in U$, and let $x(t), 0 \leq t \leq t_{0}$, be a curve in $f(U)$ from $f\left(x_{0}\right)$ to $f(u)$. Let $w(t)$ be the lift of $x(t)$ to $S^{2(n+p)+1}$ so that $w(0)=w_{0}$ and $\pi(w(t))=x(t), 0 \leq t \leq t_{0}$.

We know that for $0 \leq t \leq t_{0}$ we have

$$
\tilde{\nabla}_{\vec{x}(t)} \xi_{j}=\pi_{*}\left(\nabla_{\vec{w}(t)}^{\prime} \xi_{j}^{\prime}\right) \quad \text { for } 1 \leq j \leq p .
$$

We also know

$$
\tilde{\nabla}_{\vec{x}(t)} \xi_{j}=-A_{\xi_{j}}(\vec{x}(t))+\nabla_{\vec{x}(t)}^{\perp} \xi_{j} .
$$

For $j>k$, however, $A_{\xi_{j}}=0$ and

$$
\nabla_{\vec{x}(t)}^{\lrcorner} \xi_{j} \in \operatorname{span}\left\{\xi_{m}, J \xi_{m} \mid k+1 \leq m \leq p\right\},
$$

and thus

$$
\tilde{\nabla}_{\vec{x}(t)} \xi_{j} \in \operatorname{span}\left\{\xi_{m}, J \xi_{m} \mid k+1 \leq m \leq p\right\} .
$$

A similar result holds for $\tilde{\nabla}_{\vec{x}(t)} J \xi_{j}$. If we let

$$
V_{t}=\operatorname{span}\left\{\xi_{m}^{\prime}(w(t)), i \xi_{m}^{\prime}(w(t)) \mid k+1 \leq m \leq p\right\},
$$

then by the isomorphism $\pi_{*}$, we have for each $t$,

$$
\nabla_{\vec{w}(t)}^{\prime} \xi_{j}^{\prime} \text { and } \nabla_{\vec{w}(t)}^{\prime} i \xi_{j}^{\prime} \in V_{t}
$$


Since $g\left(w(t), \xi_{j}^{\prime}\right)=0$, for $0 \leq t \leq t_{0}$, we have $D_{\vec{w}(t)} \xi_{j}^{\prime}=\nabla_{\vec{w}(t)}^{\prime} \xi_{j}^{\prime}$, where $D$ is the Euclidean covariant derivative in $C^{n+p+1}$.

This fact and (45) imply that for all $t$, and for $k+1 \leq j \leq p$,

$$
D_{\vec{w}(t)} \xi_{j}^{\prime} \text { and } D_{\vec{w}(t)} i \xi_{j}^{\prime} \in V_{t} \text {. }
$$

Thus $V_{t}$ is a parallel Euclidean subspace along $w(t)$, i.e. for each $t, V_{t}$ is parallel to $V_{w_{0}}$ in the sense of Euclidean parallelism.

For each $t$, let $W_{t}$ be the $2(n+k+1)$-dimensional real affine space through $w(t)$ which is orthogonal to $V_{t}$. Since $V_{t}$ is parallel to $V_{w_{0}}$ for each $t, W_{t}$ is parallel to $W_{w_{0}}$ for each $t$, in the Euclidean sense of parallelism. However, for each $t,-w(t) \in W_{t}$, and thus $W_{t}$ passes through the origin for each $t$. Hence we conclude $W_{t}=W_{w_{0}}$ for $0 \leq t \leq t_{0}$.

Since $\vec{w}(t)$ is orthogonal to $V_{t}$ for all $t$, we have $\vec{w}(t) \in W_{t}=W_{w_{0}}$. Since $w(0) \in W_{w_{0}}$, this shows that $w(t) \in W_{w_{0}}$ for all $t$; and so $w(t) \in W_{w_{0}}$ $\cap S^{2(n+p)+1}=S^{2(n+k)+1}$ for $0 \leq t \leq t_{0}$. Applying $\pi$, we get $x(t) \in P^{(n+k)}(C)$ for all $t$. In particular, $f(u)=x\left(t_{0}\right) \in P^{n+k}(C)$. Since $u \in U$ was arbitrary, we have shown $f(U) \subset P^{n+k}(C)$.

To prove the global result we use the connectedness of $M^{n}$. Let $U_{1}$, $U_{2}$ be co-ordinate neighborhoods of $M^{n}$ such that $U_{1} \cap U_{2} \neq \phi$. We have shown above that there exist 2 totally geodesic $(n+k)$-dimensional submanifolds of $P^{n+p}(C)$, call them $P_{1}^{n+k}$ and $P_{2}^{n+k}$, such that $f\left(U_{1}\right) \subset P_{1}^{n+k}$ and $f\left(U_{2}\right) \subset P_{2}^{n+k}$.

Suppose $P_{1}^{n+k} \neq P_{2}^{n+k}$. Then, $P_{1}^{n+k} \cap P_{2}^{n+k}=P^{n+k-1}$, a totally geodesic $(n+k-1)$-dimensional submanifold of $P^{n+p}(C)$, and $f\left(U_{1} \cap U_{2}\right) \subset P^{n+k-1}$. This implies that for $z \in U_{1} \cap U_{2}$, the first normal space $N_{1}(z)$ has dimension $k-1$. This contradicts the assumption that $N_{1}(x)$ has constant dimension $k$ on $M^{n}$. Thus we conclude $P_{1}^{n+k}=P_{2}^{n+k}=P^{n+k}(C)$. Using this, one easily proves from the connectedness of $M^{n}$ that $f\left(M^{n}\right) \subset P^{n+k}(C)$.

Q.E.D.

Now Propositions 7, 8, and 9 combine to imply that under the hypotheses of Theorem 2, $f\left(M^{n}\right) \subset P^{n+1}(C)$, a totally geodesic $(\mathrm{n}+1)$ dimensional submanifold of $P^{n+p}(C)$. The proof of Theorem 2 then follows from Remark 1.

Section 5-The Special Case $Q^{n} \subset P^{n+1}(C)$.

In this section we make a detailed study of the case $Q^{n} \subset P^{n+1}(C)$. The main results are contained in Theorem 3 . We first discuss some 
necessary preliminaries.

Consider $C^{n+2}$ with natural basis $e_{0}, \cdots, e_{n+1}$. We denote by $H(z, w)$ the complex bi-linear form defined by

$$
H(z, w)=\sum_{k=0}^{n+1} z^{k} w^{k}, \quad \text { where } z=\sum_{k=0}^{n+1} z^{k} e_{k} \text { and } w=\sum_{k=0}^{n+1} w^{k} e_{k} .
$$

Then $Q^{n}$ is defined as

$$
Q^{n}=\left\{\pi(z) \mid z \in S^{2(n+1)+1} \text { and } H(z, z)=0\right\},
$$

where $\pi$ is the projection from $S^{2(n+1)+1}$ to $P^{n+1}(C)$. We continue to assume that $P^{n+1}(C)$ has constant holomorphic sectional curvature 4 .

Let $q \in Q^{n}$ and $\xi$ be a unit-length vector in $T_{q}^{\perp}\left(Q^{n}\right)$. Then Smyth ([8], p. 263-265) shows that $A_{\xi}$ has the following form when diagonalized,

$$
A_{\xi}=\left[\begin{array}{cc}
I_{n} & 0 \\
0 & -I_{n}
\end{array}\right] \text {, }
$$

where again $I_{n}$ is an $n \times n$ identity matrix.

With these remarks aside, we first prove the following elementary proposition.

Proposition 10. Let $z=\sum_{k=0}^{n+1} z^{k} e_{k} \in S^{2(n+1)+1}$. Then $H(z, z)=1$ if and only if $z^{k}$ is real for $0 \leq k \leq n+1$.

Proof. $H(z, z)=\sum_{k=0}^{n+1}\left(z^{k}\right)^{2}$; and if each $z^{k}$ is real, then $H(z, z)=\|z\|^{2}$ $=1$. Conversely, suppose $H(z, z)=1$. Then letting $\bar{z}=\sum_{k=0}^{n+1} \bar{z}^{k} e_{k}$, we have

$$
|(z, \bar{z})|^{2}=\left|\sum_{k=0}^{n+1}\left(z^{k}\right)^{2}\right|=1=\|z\|^{2} \cdot\|\bar{z}\|^{2} .
$$

The Schwarz inequality for the inner product (, ) implies that (46) can be true only if $\bar{z}=c z$ for some $c \in C$.

But then since $(z, z)=1$,

$$
1=\sum_{k=0}^{n+1} z^{k} \bar{z}^{k}=\sum_{k=0}^{n+1} z^{k} c z^{k}=c \sum_{k=0}^{n+1}\left(z^{k}\right)^{2}=c .
$$

Hence $c=1$ and so $\bar{z}=z$ and $z$ is real, i.e. $z^{k}$ is real for $0 \leq k \leq n+1$.

Q.E.D.

Let $R^{n+2}$ denote the real vector space spanned by $e_{0}, \cdots, e_{n+1}$. Then $S^{n+1}$, defined by $S^{n+1}=\boldsymbol{R}^{n+2} \cap S^{2(n+1)+1}$, is an $(n+1)$-dimensional Euclidean 
sphere. The projection $\pi$ takes the antipodal points $z$ and $-z \in S^{n+1}$ onto the same point $p=\pi(z) \in P^{n+1}(C)$. This is the only identification on $S^{n+1}$ induced by $\pi$, and we see that $\pi\left(S^{n+1}\right)=P^{n+1}(R)$, a real $(n+1)$-dimensional projective space naturally embedded in $P^{n+1}(C)$. Let $p \in P^{n+1}(R)$, and let $z \in S^{n+1}$ such that $\pi(z)=p$. We define a set $S_{p}^{n}$ by

$$
S_{p}^{n}=\left\{\pi\left(\frac{x+i z}{\sqrt{2}}\right) \mid x \in S^{n+1}, g(x, z)=0\right\}
$$

One easily shows that $S_{p}^{n}$ is independent of the choice of $z$.

Proposition 11. Let $p \in P^{n+1}(R)$, then $S_{p}^{n}$ is the image of a Euclidean $n$-sphere of radius $1 / \sqrt{2}$ isometrically embedded in $P^{n+p}(C)$.

Proof. Let $z \in S^{n+1}$ such that $\pi(z)=p$. We define $\boldsymbol{R}^{n+1}$ by

$$
\boldsymbol{R}^{n+1}=\left\{w \in \boldsymbol{R}^{n+2} \mid g(z, w)=0\right\} .
$$

Let $\overline{\boldsymbol{R}}^{n+2} \equiv \boldsymbol{R}^{n+1} \times\{i z\}$ where $\{i z\}$ is the 1-dimensional real subspace spanned by the vector $i z$. Then

$$
\bar{S}^{n+1} \equiv \overline{\boldsymbol{R}}^{n+2} \cap S^{2(n+1)+1}
$$

is a Euclidean $(n+1)$-sphere of radius 1 . Then

$$
S \equiv\left\{\frac{x+i z}{\sqrt{2}} \mid x \in S^{n+1}, g(x, z)=0\right\} \subset \bar{S}^{n+1} .
$$

In fact, it is easy to see that $S$ is a small-sphere of dimension $n$ with center $i z / \sqrt{2}$ and radius $1 / \sqrt{2}$ contained in $\bar{S}^{n+1}$. One checks that no two points of $S$ are identified under the projection $\pi$. Thus $\pi$ is a one-toone isometry on $S$, and $\pi(S)=S_{p}^{n}$ is the image of a Euclidean $n$-sphere of radius $1 / \sqrt{2}$ isometrically embedded in $P^{n+p}(C)$ Q.E.D.

The following theorem describes the focal point behavior for $Q^{n} \subset P^{n+1}(C)$.

THEOREM 3. (i) The set of focal points of $Q^{n} \subset P^{n+1}(C)$ is $P^{n+1}(R)$.

(ii) Let $p \in P^{n+1}(R)$; then

$\left\{q \in Q^{n} \mid p\right.$ is a focal point of $\left.\left(Q^{n}, q\right)\right\}=S_{p}^{n}$.

Proof. To prove (i), we first show that the set of focal points of $Q^{n}$ is contained in $P^{n+1}(\boldsymbol{R})$.

Let $p \in P^{n+1}(C)$ be a focal point of $\left(Q^{n}, q\right)$ for some $q \in Q^{n}$. By 
Proposition 1, $p=F(q, r \xi)$ where $\xi$ is a unit-length vector in $T_{q}^{\perp}\left(Q^{n}\right)$ and $\cot r=\lambda$ for some eigen-value $\lambda$ of $A_{\xi}$. As we remarked at the beginning of this section, $\lambda= \pm 1$ for any such $q$ and $\xi$. Choosing the sign of $\xi$ properly we may assume $\cot r=1$, and then

$$
F\left(q, \frac{\pi}{4} \xi\right)=\pi\left(\frac{w}{\sqrt{2}}+\frac{\xi^{\prime}}{\sqrt{2}}\right) \quad \text { where } \pi(w)=q \text { and } \pi_{*}\left(\xi^{\prime}\right)=\xi .
$$

It is known (see [4], Vol. II, p. 279) that there exist unique real vectors $x, y$ of length $1 / \sqrt{2}$, with $g(x, y)=0$, such that $w=x+i y$. Then $T_{q}^{\perp}\left(Q^{n}\right)$ is spanned by $\pi_{*}(i x+y)$ and $\pi_{*}(-x+i y)$. Thus we can express $\xi^{\prime}$ as

$$
\xi^{\prime}=\cos \phi(i x+y)+\sin \phi(-x+i y) \quad \text { for some } \phi, 0 \leq \phi \leq 2 \pi .
$$

Thus $p=\pi(z)$ where

$$
\begin{aligned}
z & =\frac{1}{\sqrt{2}}(w+\cos \phi(i x+y)+\sin \phi(-x+i y)) \\
& =\frac{x}{\sqrt{2}}[(1-\sin \phi)+i \cos \phi]+\frac{y}{\sqrt{2}}[\cos \phi+(1+\sin \phi) i] .
\end{aligned}
$$

Using the defining properties of $x$ and $y$, we compute

$$
H(z, z)=-\sin \phi+i \cos \phi=e^{i(\phi+\pi / 2)} .
$$

Let $z^{\prime}=e^{-i(\phi+\pi / 2) / 2} z$; then $\pi\left(z^{\prime}\right)=p$, but

$$
H\left(z^{\prime}, z^{\prime}\right)=e^{-i(\phi+\pi / 2)} H(z, z)=1 .
$$

Thus by Proposition 10, $z^{\prime}$ is real, and so $p \in P^{n+1}(R)$.

Conversely, suppose $p=\pi(z)$ where $z \in S^{n+1}$. Let $x \in S^{n+1}$ such that $g(x, z)=0$. Let $w=(x+i z) / \sqrt{2}$. Then,

$$
H(w, w)=0, \text { and } q=\pi(w) \in Q^{n} .
$$

One easily shows that $\xi^{\prime}=(-x+i z) / \sqrt{2} \in T_{w}^{\prime}$ and $\pi_{*}\left(\xi^{\prime}\right) \in T_{q}^{\perp}\left(Q^{n}\right)$. If we let

$$
z^{\prime}=\frac{1}{\sqrt{2}}\left(\frac{x+i z}{\sqrt{2}}\right)+\frac{1}{\sqrt{2}}\left(\frac{-x+i z}{\sqrt{2}}\right)=i z
$$

then by Proposition 1, $\pi\left(z^{\prime}\right)$ is a focal point of $\left(Q^{n}, q\right)$. But $\pi\left(z^{\prime}\right)=\pi(i z)=p$, and so the proof of (i) is complete.

To prove (ii) we let $p=\pi(z)$ for $z \in S^{n+1}$. Let 


$$
S=\left\{(x+i z) / \sqrt{2} \mid x \in S^{n+1}, g(x, z)=0\right\}
$$

and

$$
T=\left\{q \in Q^{n} \mid p \text { is a focal point of }\left(Q^{n}, q\right)\right\} .
$$

By definition $S_{p}^{n}=\pi(S)$, and in the above proof of (i) we showed that $S_{p}^{n} \subset T$. To complete the proof of (ii), we show $T \subset S_{p}^{n}$.

Suppose $q \in T$. Let $w \in S^{2(n+1)+1}$ such that $\pi(w)=q$. Then $w=$ $(x+i y) / \sqrt{2}$ for a unique choice of $x, y \in S^{n+1}$ such that $g(x, y)=0$. By (i) we know $p \in P^{n+1}(R)$, so there is $z \in S^{n+1}$ such that $\pi(z)=p$. We first show

$$
z=\cos \alpha x+\sin \alpha y \quad \text { for some } \alpha, 0 \leq \alpha \leq 2 \pi .
$$

We know that $T_{q}^{\perp}\left(Q^{n}\right)$ is spanned by

$$
\pi_{*}\left(\frac{-x+i y}{\sqrt{2}}\right) \text { and } \pi_{*}\left(\frac{i x+y}{\sqrt{2}}\right) .
$$

By Proposition 1, any focal point of $\left(Q^{n}, q\right)$ can be expressed as $\pi(u)$ where

$$
u=\frac{1}{\sqrt{2}}\left(\frac{x+i y}{\sqrt{2}}\right)+\frac{1}{\sqrt{2}}\left(\cos \phi\left(\frac{-x+i y}{\sqrt{2}}\right)+\sin \phi\left(\frac{i x+y}{\sqrt{2}}\right)\right)
$$

for some $\phi, 0 \leq \phi \leq 2 \pi$.

Since $\pi(z)=p$ is a focal point of $\left(Q^{n}, q\right)$, we must have $z=e^{i \beta} u$ for some $u$ as in (47), and for some $\beta, 0 \leq \beta \leq 2 \pi$. This implies that $z$ is a real linear combination of $x, y, i x$ and $i y$. Since $x, y$ and $z$ are all real, we must have

$$
z=\cos \alpha x+\sin \alpha y \quad \text { for some } \alpha, 0 \leq \alpha \leq 2 \pi .
$$

Consider $w^{\prime}=(\sin \alpha+i \cos \alpha)[(x+i y) / \sqrt{2}]$. Then $\pi\left(w^{\prime}\right)=\pi(w)=$ $q$. But from (48) we see

$$
\begin{aligned}
w^{\prime} & =\frac{1}{\sqrt{2}}[(\sin \alpha x-\cos \alpha y)+i(\cos \alpha x+\sin \alpha y)] \\
& =\frac{1}{\sqrt{2}}[(\sin \alpha x-\cos \alpha y)+i z]
\end{aligned}
$$

Thus $w^{\prime} \in S$, and $q \in \pi(S)=S_{p}^{n}$. This is true for any $q \in T$, and we have $T \subset S_{p}^{n}$.

Q.E.D. 


\section{BIBLIOGRAPHY}

[1] L. Bers, Introduction to Several Complex Variables, Courant Institute of Mathematical Sciences, New York University, New York, 1964.

[2] E. Cartan, Lecons sur la geometrie des espaces de Riemann, deuxième edition, Gauthier-Villars, Paris, 1946.

[ 3 ] J. Erbacher, Reduction of the codimension of an isometric immersion, J. Differential Geometry 5 (1971), 333-340.

[4] S. Kobayashi and K. Nomizu, Foundations of Differential Geometry, Vol. I-II, John Wiley and Sons, Inc., New York, 1963, 1969.

[5] J. Milnor, Morse Theory, Ann. of Math. Studies, No. 51, Princeton University Press, 1963.

[6] K. Nomizu and L. Rodriguez, Umbilical Submanifolds and Morse Functions, Nagoya Math. J. 48 (1972), 197-201.

[ 7 ] K. Nomizu and B. Smyth, Differential Geometry of Complex Hypersurfaces II, J. Math. Soc. Japan 20 (1968), 498-521.

[ 8 ] B. Smyth, Differential Geometry of Complex Hypersurfaces, Ann. of Math. 85 (1967), 246-266.

Vassar College 